\title{
ROBUST NONLINEAR OBSERVER DESIGN FOR ACTUATOR FAULT DETECTION IN DIESEL ENGINES
}

\author{
Boulaid BOULKROUNE ${ }^{*, * *}$, Issam DJEMILI ${ }^{*, * *}$, Abdel AITOUCHE ${ }^{*, * *}$, \\ VINCENT COCQUEMPOT ${ }^{* *}$ \\ ${ }^{*}$ HEI, Engineer School, 13, rue de Toul, 59046, Lille, France \\ e-mail: boulkrb@gmail.com, \{issam.djemili, abdel.aitouche\}@hei.fr \\ ** Automatic Control Laboratory (LAGIS) \\ Lille 1 University, Rue Paul Langevin, 59655, Villeneuve d'Ascq, France \\ e-mail: vincent. cocquempotauniv-lille1.fr
}

\begin{abstract}
This paper is concerned with actuator fault detection in nonlinear systems in the presence of disturbances. A nonlinear unknown input observer is designed and the output estimation error is used as a residual for fault detection. To deal with the problem of high Lipschitz constants, a modified mean-value theorem is used to express the nonlinear error dynamics as a convex combination of known matrices with time-varying coefficients. Moreover, the disturbance attenuation is performed using a modified $H_{\infty}$ criterion. A sufficient condition for the existence of an unknown input observer is obtained using a linear matrix inequality formula, and the observer gains are obtained by solving the corresponding set of inequalities. The advantages of the proposed method are that no a priori assumption on the unknown input is required and that it can be applied to a large class of nonlinear systems. Performances of the proposed approach are shown through the application to a diesel engine model.
\end{abstract}

Keywords: diesel engine, fault diagnosis, nonlinear unknown input observers, LMI.

\section{Introduction}

In automotive industry, on-board diagnosis of engines has become increasingly important because of environmentally based legislative regulations such as OBDII (On-Board Diagnostics-II) (CARB, 1993). Model-based diagnosis of automotive engines has been considered in earlier papers (see, e.g., Gertler et al., 1995; Nyberg and Perkovic, 1998). However, the engines investigated in these previous works were all gasoline-fuelled and did not include Exhaust Gas Recirculation (EGR) and Variable Geometry Turbocharged (VGT). Both these components make the diagnosis problem significantly more difficult since the air flow through the EGR-valve and also the exhaust side of the engine have to be taken into account. An interesting approach to model-based air-path faults detection for an engine which includes EGR and VGT can be found in the works of Nyberg (2002) as well as Nyberg and Sutte (2004). By using several models in parallel, where each one is sensitive to one kind of fault, predicted outputs are compared and a diagnosis is provided. In particular, the hypothesis test methodology proposed by Nyberg (2002) deals with multi-fault detection in an well as air-path system. Nyberg and Sutte (2004) propose an extended adaptive Kalman filter to find which faulty model best matches with measured data, then a structured hypothesis allows going back to the faults. Recently, a structural analysis for the air path of an automotive diesel engine has been developed in order to study the monitorability of the system (Djemili et al., 2011b).

Other approaches to detect intake leakages in diesel engines based on adaptive observers were proposed by Ceccarelli et al. (2009a; 2009b) and recently by Djemili et al. (2011a). Note that in all these approaches the leakage size is assumed to be constant. Furthermore, few works dealing with actuator faults detection in diesel engines with EGR and VGT are proposed in the literature due to the complexity of the system. Indeed, the engine models have high nonlinearities with large Lipschitz constants. In this paper, the engine model is divided into two parts: a Linear Parameter Varying (LPV) part and a nonlinear part. Notice that disturbances are also 
considered in this work. For the sake of fault detection and diagnosis, each residual is designed to be sensitive to only one fault and insensitive to the other faults that can affect the system, which will be considered unknown inputs. Therefore, the proposed approach takes into account the presence of unknown inputs, which makes residual design much more complicated.

Designing Unknown Input Observers (UIOs) has received considerable attention in the literature. The challenge in UIO design is to construct an observer such that it can estimate the states of the system considered asymptotically without any knowledge of the unknown input. If this issue in the linear case is well solved, it remains open in the nonlinear one. The first unknown input observers dedicated to linear systems were proposed by Kudva et al. (1980), Hou and Muller (1992), Darouach et al. (1994), as well as Hui and Żak (2005). Necessary and sufficient conditions of the existence of the UIO have been well established.

For the nonlinear case, new sufficient conditions formulated in terms of the Linear Matrix Inequality (LMI) are given by Chen and Saif (2006). The major advantage of this approach lies in its simplicity. Indeed, here sufficient conditions of the existence of Nonlinear Unknown Input Observers (NUIOs) are formulated only in terms of LMIs, avoiding a combination of LMIs with Linear Matrix Equalities (LMEs) as proposed by Yaz and Azemi (1998). Notice that it is not an easy task to find a solution satisfying together LMIs and LMEs.

The approach proposed by Chen and Saif (2006) will be extended in this paper to cover a wide class of nonlinear systems that cannot be treated by this approach as well as nonlinear systems with large Lipschitz constants. Several approaches have tried to extend unknown input observer design for linear systems to nonlinear ones. The first works were dedicated to UIOs for bilinear systems (e.g., Saif, 1993). Other approaches based on the transformation of the nonlinear system into a canonical form are proposed by Ding et al. (1990) as well as Seliger and Frank (1991). A limitation of these approaches is that the required state transformation does not exist for all nonlinear systems but only for a limited class. Zemouche and Boutayeb (2009) presented a UIO design method for a class of nonlinear systems based on the modified $H_{\infty}$ criterion. The state of the system and the unknown inputs are estimated jointly despite the presence of the disturbances in the dynamics and the output of the system.

The Mean Value Theorem (MVT) is used to deal with the problem of a nonlinear system having a large Lipschitz constant. However, this method is computationally demanding since the number of LMIs that have to be solved depends on the number of nonlinearities and the dimension of the system. To overcome this difficulty, an approach based on the Modified Mean Value Theorem (MMVT) is proposed by Phanomchoeng et al.
(2011). It expresses the nonlinear error dynamics as a convex combination of known matrices with time-varying coefficients, which significantly reduces the number of LMIs to be solved.

In this paper, the approach based on the MMVT will be extended to the proposed class of nonlinear systems, namely, nonlinear parameter varying ones. For the fault detection purpose, several observer-based approaches are proposed in the literature for nonlinear systems (see, e.g., Chen et al., 2011). The highlights of this work include the following:

- the proposed method concerns a more general class of nonlinear parameter varying systems with unknown inputs for which the literature in this field is very limited;

- the problem of robust fault detection and isolation for nonlinear parameter varying systems with unknown inputs and disturbances is investigated. The advantage of the proposed method is that no a priori assumption on the unknown input is required;

- sufficient conditions of the existence of the NUIO are formulated, in an elegant way, in terms of linear matrix inequalities;

- the MMVT approach is used for reducing the number of LMIs to be solved;

- the performances of the proposed approach are shown using the professional diesel engine simulator AMEsim(LMS) in co-simulation with the Simulink software.

This paper is organized as follows. Problem formulation and preliminaries are presented in Section 2. Section 3 is devoted to the design of a nonlinear unknown input observer. A description of the diesel engine model is presented in Section 4. The proposed approach is successfully validated using an advanced diesel engine professional simulator in Section 5. A conclusion ends the paper.

\section{Problem formulation and preliminaries}

Some notation and definitions that will be used throughout this paper are first introduced. $\mathbb{R}$ denotes the set of real numbers. The set of $p \times q$ real matrices is denoted by $\mathbb{R}^{p \times q}$. $A^{T}$ and $A^{-1}$ represent the transpose of matrix $A$ and its left inverse (assuming that $A$ has a full column rank), respectively. $I_{r}$ represents the identity matrix of dimension $r .0$ (in bold character) is a zero matrix with appropriate dimensions. $(\star)$ is used for the blocks induced by symmetry. $\|\cdot\|$ represents the usual Euclidean norm. $\mathfrak{L}_{2}$ denotes the Lebesgue space. 
Let us consider a general class of nonlinear systems described by the following equations:

$$
\begin{aligned}
\dot{x}= & A(\rho) x+B_{g} g(v, y, u)+\varphi(x, u)+B_{d} d \\
& +B_{f} f+B_{w} w \\
y= & C x+D_{w} w
\end{aligned}
$$

with $A(\rho)=\sum_{j=1}^{n_{\rho}} \rho_{j} A_{j}$. Here $x \in \mathbb{R}^{n_{x}}$ is the state vector, $u \in \mathbb{R}^{n_{u}}$ is the control input vector which is assumed to be bounded, $d \in \mathbb{R}^{n_{d}}$ represents the unknown input vector, $f$ is the actuator fault vector, $y \in \mathbb{R}^{n_{y}}$ is the output vector, $w \in \mathbb{R}^{n_{w}}$ is the vector of disturbances and $v \in \mathbb{R}^{n_{v}}$ is the vector of measurable signals. $A_{j}$, with $j=1, \ldots, n_{\rho}, B_{g}, B_{d}, B_{f}, B_{w}, C$ and $D_{w}$ are constant matrices with appropriate dimensions.

Without loss of generality, $B_{d}$ and $B_{f}$ are assumed to be of full column rank. The functions $g(v, y, u)$ and $\varphi(x, u)$ are nonlinear. The function $\varphi(x, u)$ is assumed to be Lipschitz in $x$ and differentiable. The weighting functions $\rho_{j}$ are assumed to be known and depend on measurable variables. It is supposed that

$$
\sum_{j=1}^{n_{\rho}} \rho_{j}=1, \rho_{j} \geq 0, \quad \forall j \in\left\{1, \ldots, n_{\rho}\right\} .
$$

For manipulating the nonlinear function $\varphi(x, u)$, the MMVT presented for a general vector function $\varphi(x, u)$ by Phanomchoeng et al. (2011) is applied in this work. It will be used in Section 3 to express the nonlinear error dynamics as a convex combination of known matrices with time-varying coefficients. The main principle of the MMVT is presented in the following theorem.

Theorem 1. (Phanomchoeng et al., 2011) Let the canonical basis of the vectorial space $\mathbb{R}^{s}$ for all $s \geq 1$ be defined by

$$
\begin{array}{r}
E_{s}=\left\{e_{s}(i) \mid e_{s}(i)=(\underbrace{0, \ldots, 0, \overbrace{1}^{i-t h}, 0, \ldots, 0}_{s \text { components }})^{T},\right. \\
i=1, \ldots, s\} .
\end{array}
$$

Let $\varphi(x): \mathbb{R}^{n} \rightarrow \mathbb{R}^{n}$ be a vector function continuous on $[a, b] \in \mathbb{R}^{n}$ and differentiable on any convex hull of the set $(a, b)$. For $s_{1}, s_{2} \in[a, b]$, there exist $\delta_{i j}^{\max }$ and $\delta_{i j}^{\min }$ for $i=1, \ldots, n$ and $j=1, \ldots, n$ such that

$$
\begin{aligned}
& \varphi\left(s_{2}\right)-\varphi\left(s_{1}\right) \\
& =\left[\left(\sum_{i, j=1}^{n, n} H_{i j}^{\max } \delta_{i j}^{\max }\right)+\left(\sum_{i, j=1}^{n, n} H_{i j}^{\min } \delta_{i j}^{\min }\right)\right] \\
& \quad \times\left(s_{2}-s_{1}\right) \\
& \quad \delta_{i j}^{\max }, \quad \delta_{i j}^{\min } \geq 0, \delta_{i j}^{\max }+\delta_{i j}^{\min }=1,
\end{aligned}
$$

where

$$
\begin{aligned}
h_{i j}^{\max } & \geq \max \left(\frac{\partial \varphi_{i}}{\partial x_{j}}\right) \quad \forall x \in(a, b), \\
h_{i j}^{\min } & \geq \min \left(\frac{\partial \varphi_{i}}{\partial x_{j}}\right), \quad \forall x \in(a, b), \\
H_{i j}^{\max } & =e_{n}(i) e_{n}^{T}(j) h_{i j}^{\max }, \\
H_{i j}^{\min } & =e_{n}(i) e_{n}^{T}(j) h_{i j}^{\min } .
\end{aligned}
$$

The proof of this theorem is given by Phanomchoeng et al. (2011).

In our case, the nonlinear function $\varphi$ depends on the state vector $x$ and also on the known input $u$. The previous theorem is applicable in our case without changes or modifications since $u$ is bounded.

The aim now is to design a nonlinear unknown input observer which can reconstruct the state behavior without any knowledge of the unknown inputs.

\section{Robust nonlinear unknown input observer design}

In this section, inspired by Chen and Saif (2006) we present a Nonlinear Unknown Input Observer (NUIO) design method for the class of Lipschitz nonlinear systems given by (1). The discussed system (1) represents a large class of nonlinear systems with a large or small Lipschitz constant. It is well known that most of the existing NUIO design approaches for this class of systems fail to provide a solution when the Lipschitz constant is large. In this context the modified mean value theorem presented by Phanomchoeng et al. (2011) will be used. The sufficient existence conditions for the observer design are formulated in terms of LMIs.

3.1. NUIO design. The observer considered for the system (1) is given by

$$
\begin{aligned}
& \dot{z}=N(\rho) z+G g(v, y, u)+M \varphi(\hat{x}, u)+L(\rho) y, \\
& \hat{x}=z-E y,
\end{aligned}
$$

with

$$
\begin{aligned}
& N(\rho)=\sum_{j=1}^{n_{\rho}} \rho_{j} N_{j}, \\
& L(\rho)=\sum_{j=1}^{n_{\rho}} \rho_{j} L_{j} .
\end{aligned}
$$

All $N_{j}$ are stable matrices. $\hat{x}$ represents the state estimation vector of $x$. Matrices $N, G, M, L$ and $E$ are the observer gains and matrices which must be determined such that $\hat{x}$ converges asymptotically to $x$ when $f=0$ and in the absence of disturbances. Notice that the index $\rho$ is omitted where it is not necessary in order to keep the notation clear and easy to read. 
Let us define the state estimation error as

$$
e(t)=\hat{x}(t)-x(t) .
$$

Using (1b) and (5b), the state estimation error $e(t)$ can be expressed as

$$
e(t)=z-\left(I_{n_{x}}+E C\right) x(t)-E D_{w} w .
$$

By setting $M=I_{n_{x}}+E C$, the error dynamics can be given by

$$
\begin{aligned}
\dot{e}= & N e+\left(G-M B_{g}\right) g(v, y, u)+(N M+L C-M A) x \\
& -M B_{d} d+M \tilde{\varphi}-M B_{f} f+\left(K D_{w}-M B_{w}\right) w \\
& -E D_{w} \dot{w},
\end{aligned}
$$

with $\tilde{\varphi}=\varphi(\hat{x}, u)-\varphi(x, u)$ and $K=L+N E$. Now, if the following matrix equations are satisfied:

$$
\begin{aligned}
N(\rho) & =M A(\rho)-K(\rho) C, \\
L(\rho) & =K(\rho)\left(I_{n_{x}}+C E\right)-M A(\rho) E, \\
G & =M B_{g}, \\
M & =I_{n_{x}}+E C, \\
M B_{d} & =0,
\end{aligned}
$$

$e(t)$ goes to zero asymptotically when $f=0$ and is invariant with respect to the unknown input $d(t)$.

To obtain matrices $E, M, G, K, N$ and $L$, the following steps should be followed:

- First, the conditions (8d) and (8e) are equivalent to $E C B_{d}=-B_{d}$. One necessary condition to have $E C B_{d}=-B_{d}$ is that $C B_{d}$ must be of full column rank since $B_{d}$ is so. If $C B_{d}$ is of full column rank, then all possible solutions of $E C B_{d}=-B_{d}$ can be expressed as follows (Chen and Saif, 2006):

$$
E=U+Y V,
$$

with

$$
U=-B_{d}\left(C B_{d}\right)^{\dagger}
$$

and

$$
V=\left(I_{n_{y}}-C B_{d}\left(C B_{d}\right)^{\dagger}\right),
$$

where $Y$ can be any compatible matrix and $X^{\dagger}=$ $\left(X^{T} X\right)^{-1} X^{T}$.

- Then, the matrix $M$ is obtained by substituting $E$ in $8 \mathrm{c}$.

- Using (8c) and (8d), the matrix $G$ is obtained.

- The matrix $K$ is determined such that the matrix $M A(\rho)-K(\rho) C$ is stable. The gain $K$ will be obtained from the solution of the LMIs given in Theorem 2.
- Finally, by substituting $K, M$ and $E$ in (8b), the gain $L$ will be obtained.

Then, the error dynamics become

$$
\begin{aligned}
\dot{e}= & (M A-K C) e+M \tilde{\varphi}+\left(K D_{w}-M B_{w}\right) w \\
& -E D_{w} \dot{w}-M B_{f} f .
\end{aligned}
$$

As explained before, if the conditions (8a)-(8e) hold, the estimation error $e$ without fault $(f=0)$, named $\bar{e}$, tends asymptotically to zero in the absence of disturbances. Thus, the dynamics of $\bar{e}$ are expressed as

$$
\begin{aligned}
\dot{\bar{e}}= & (M A-K C) \bar{e}+M \tilde{\varphi}+\left(K D_{w}-M B_{w}\right) w \\
& -E D_{w} \dot{w} .
\end{aligned}
$$

3.2. Stability and convergence analysis. In order to minimize the effect of disturbances on the estimation error $\bar{e}$, the $H_{\infty}$ performance criterion can be used. However, the presence of the term $\dot{w}$ makes the task difficult because it should be discarded from the derivative of the Lyapunov function, as we shall see later. Another solution is to add a negative term depending of $\dot{w}^{T} \dot{w}$ as proposed by Zemouche and Boutayeb (2009). This solution needs to modify the classical $H_{\infty}$ criterion.

The modified $H_{\infty}$ estimation problem consists in computing the matrices $N$ and $L$ such that

$$
\begin{aligned}
& \lim _{t \rightarrow \infty} \bar{e}(t)=0 \quad \text { for } w(t)=0 \quad \text { and } f(t)=0, \\
& \|\bar{e}\|_{\mathfrak{L}_{2}^{n_{x}}} \leq \gamma_{1,2}\|w\|_{1,2}^{r} \quad \text { for } \quad w(t) \neq 0, \bar{e}(0)=0 .
\end{aligned}
$$

Then, to satisfy 12a -12b, it is sufficient to propose a Lyapunov function $\Upsilon$, where

$$
\Gamma=\dot{\Upsilon}+\bar{e}^{T} \bar{e}-\gamma_{1,2}^{2} w^{T} w-\gamma_{1,2}^{2} \dot{w}^{T} \dot{w}<0,
$$

$\Upsilon=\bar{e}^{T} P \bar{e}$ with $P$ a positive definite symmetric matrix. From (11), $\Gamma$ is given by

$\Gamma$

$$
\begin{aligned}
= & \bar{e}^{T}\left((M A-K C)^{T} P+P(M A-K C)\right) \bar{e} \\
& +\bar{e}^{T} P M \tilde{\varphi}+(M \tilde{\varphi})^{T} P \bar{e}+\bar{e}^{T} P\left(K D_{w}-M B_{w}\right) w \\
& +w^{T}\left(K D_{w}-M B_{w}\right)^{T} P \bar{e}-\bar{e}^{T} P E D_{w} \dot{w} \\
& -\dot{w}^{T}\left(E D_{w}\right)^{T} P \bar{e}+\bar{e}^{T} \bar{e}-\gamma_{1,2}^{2} w^{T} w-\gamma_{1,2}^{2} \dot{w}^{T} \dot{w} .
\end{aligned}
$$

Now, we can give a sufficient condition under which the observer given by (5) is an NUIO.

Theorem 2. The observer error $\bar{e}(t)$ converges asymptotically towards zero if there exists matrices $\bar{K}_{k}, \bar{Y}, a$ positive definite symmetric matrix $P$ and a positive scalar 
$\mu$ such that the following LMIs are satisfied:

$$
P>0, \quad(15 a)
$$

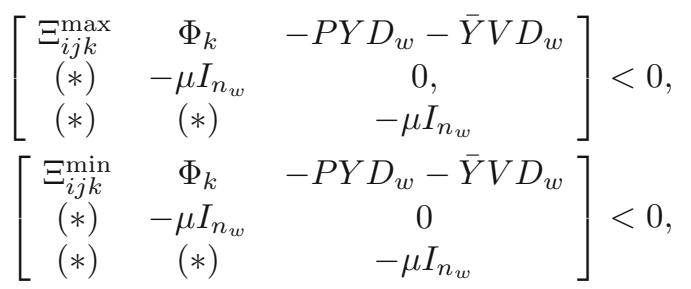
where

$$
\forall i=1, \ldots, n, j=1, \ldots, n \text { and } k=1, \ldots, n_{\rho},
$$

$$
\begin{aligned}
\Phi_{k}= & -\bar{K}_{k} D_{w}+P\left(I_{n_{x}}+U C\right) B_{w}+\bar{Y} V C B_{w} \\
\Xi_{i j k}^{\max }= & {\left[\left(I_{n_{x}}+U C\right)\left(A_{k}+\bar{H}_{i j}^{\max }\right)\right]^{T} P } \\
& +P\left(I_{n_{x}}+U C\right)\left(A_{k}+\bar{H}_{i j}^{\max }\right) \\
& -C^{T} \bar{K}_{k}^{T}-\bar{K}_{k} C+\left(A_{k}+\bar{H}_{i j}^{\max }\right)^{T} C^{T} V^{T} \bar{Y}^{T} \\
& +\bar{Y} V C\left(A_{k}+\bar{H}_{i j}^{\max }\right)+I_{n_{x}}, \\
\Xi_{i j k}^{\min }= & {\left[\left(I_{n_{x}}+U C\right)\left(A_{k}+\bar{H}_{i j}^{\min }\right)\right]^{T} P } \\
& +P\left(I_{n_{x}}+U C\right)\left(A_{k}+\bar{H}_{i j}^{\min }\right)-C^{T} \bar{K}_{k}^{T} \\
& -\bar{K}_{k} C+\left(A_{k}+\bar{H}_{i j}^{\min }\right)^{T} C^{T} V^{T} \bar{Y}^{T} \\
& +\bar{Y} V C\left(A_{k}+\bar{H}_{i j}^{\min }\right)+I_{n_{x}}, \\
\bar{H}_{i j}^{\max }= & Z_{H} H_{i j}^{\max }, \quad \bar{H}_{i j}^{\min }=Z_{H} H_{i j}^{\min },
\end{aligned}
$$

with $Z_{H}=n \times n$. Solving the LMIs (15a)-(15c) leads to finding matrices $P, \bar{Y}$ and $\bar{K}_{k}$. The matrices $K_{k}$ and $Y$ can be obtained from $K_{k}=P^{-1} \bar{K}_{k}$ and $Y=P^{-1} \bar{Y}$. The other matrices $N$ and $L$ can then be deduced easily from Eqns. (8a) and (8b), respectively.

Proof. The proof is given in Appendix A.

Notice that if there exist components of $\frac{\partial \varphi_{i}}{\partial x_{j}}$ which equal 0 , the scaling factor $\sum_{i, j=1}^{n, n}\left(\delta_{i j}^{\max }+\delta_{i j}^{\min }\right)$ is less than 1. Consequently, the scaling factor $\bar{Z}_{H}$ must be redefined as follows:

$$
\begin{aligned}
& \bar{Z}_{H}=\sum_{i, j=1}^{n, n}\left(\delta_{i j}^{\max }+\delta_{i j}^{\min }\right)=n \times n-n_{0}, \\
& \frac{\sum_{i, j=1}^{n, n}\left(\delta_{i j}^{\max }+\delta_{i j}^{\min }\right)}{\bar{Z}_{H}}=1,
\end{aligned}
$$

where $n_{0}$ is the number of terms in $\frac{\partial \varphi_{i}}{\partial x_{j}}$ that equal zero.

If the conditions (8a)-(8e) hold, the estimation error $e$ and a residual $r$ can be expressed as

$$
\begin{aligned}
\dot{e} & =\bar{N} e+\left(K D_{w}-M B_{w}\right) w-E D_{w} \dot{w}-M B_{f} f \\
r & =C e+D_{w} w
\end{aligned}
$$

with

$$
\begin{aligned}
\bar{N} & =N+M\left[\left(\sum_{i, j=1}^{n, n} H_{i j}^{\max } \delta_{i j}^{\max }\right)\right. \\
& \left.+\left(\sum_{i, j=1}^{n, n} H_{i j}^{\min } \delta_{i j}^{\min }\right)\right] .
\end{aligned}
$$

As demonstrated before, the convergence of the estimation error $e$ without a fault is ensured by the gain $K$ obtained from Theorem 2 Notice also that the disturbance effect is attenuated using the $H_{\infty}$ criterion. Thus, the mean of the error vector $e$ will be close to zero in fault free operation mode, while its mean value will be significantly different from zero in the presence of a fault. Note that, in order to ensure the detection and isolation of the fault $f$, the following condition has to be satisfied:

$$
\operatorname{rank}\left(M B_{f}\right)=\operatorname{rank}\left(B_{f}\right) .
$$

\section{Engine description}

The diesel engine considered in this paper is a four-cylinder engine with a high-pressure EGR circuit and a VGT as described by Albrecht et al. (2006). A principle illustration scheme is shown in Fig. 11 The air path system consists of two parts: the turbocharger and exhaust gas recirculation. The turbocharger is a turbine driven by the exhaust gas and connected via a common shaft to the compressor, which compresses the air in the intake. Exhaust gas recirculation allows recirculating gas from the exhaust manifold to the intake manifold. The recirculation of the exhaust gas through an EGR valve into the intake manifold where it dilutes the incoming fresh air is a well-established and efficient means of reducing in-cylinder $N O_{x}$ emissions.

The mean value engine modelling approach is the most widely considered approach in the literature (Kao and Moskwa, 1995). It uses temporal and spatial averages of relevant temperatures, pressures and mass flow rates. In this work, the used model is based on principles described by Heywood (1992) as well as Nyberg and Perkovic (1998). For diagnosing the faults of interest, it is not necessary to include the compressor and the Charge-Air Cooler (CAC) in the model as shown by Nyberg and Perkovic (1998). The reason is that no fault in the compressor or the CAC is considered, and also the mass-flow and the temperature after the CAC are known variables because they are measured by the production sensors.

The engine is equipped with sensors measuring the in-flowing air $W_{\mathrm{HFM}}$, temperature after the CAC $T_{C A C}$, inlet-manifold pressure $P_{\text {Inlet }}$, exhaust-pressure $P_{\text {Exh }}$ and exhaust-temperature $T_{\text {Exh }}$. The control inputs are the injected fuel $W_{\text {Fuel }}$, the turbine vane position 


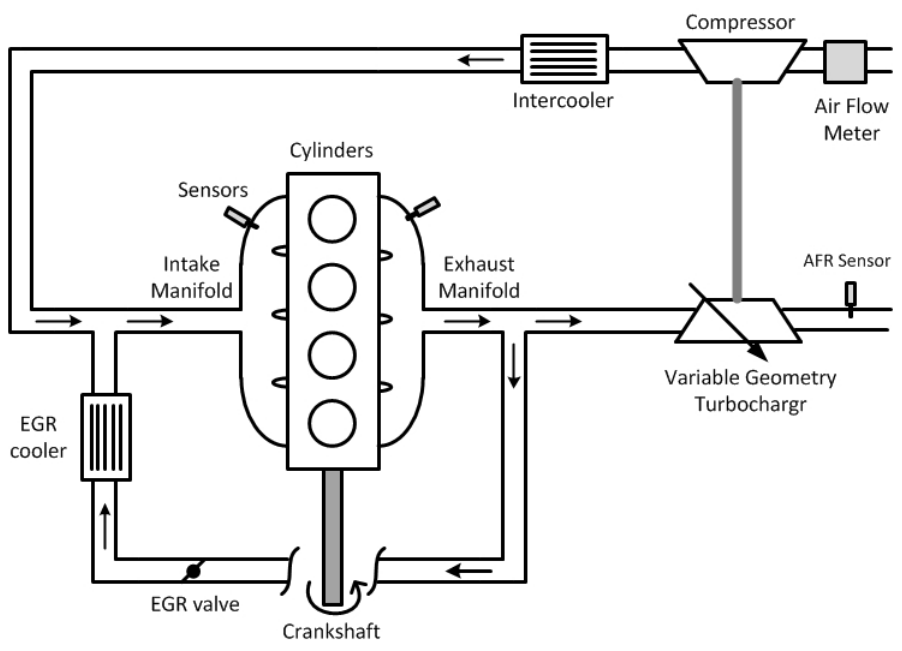

Fig. 1. Schematic picture of the air-intake system.

$X_{\mathrm{VGT}}$, and the EGR-valve position determining the valve opening-area $A_{\mathrm{EGR}}$. follows:

The diesel engine model considered is expressed as

$$
\begin{aligned}
\dot{P}_{\text {Inlet }}= & \frac{1}{V_{\text {Inlet }}}\left(\frac{R_{\text {Air }} c_{p, \text { Air }}}{c_{v, \text { Air }}} W_{\mathrm{HFM}} T_{\mathrm{CAC}}\right. \\
& +\frac{R_{\mathrm{Exh}} c_{p, \text { Exh }}}{c_{v, \text { Exh }}} W_{\mathrm{EGR}} T_{\mathrm{EGR}} \\
& \left.-\frac{R_{\text {Inlet }} c_{p, \text { Inlet }}}{c_{v, \text { Inlet }}} W_{\text {Inlet }} T_{\text {Inlet }}\right), \\
\dot{m}_{\mathrm{Air}}= & W_{\mathrm{HFM}}-\frac{m_{\mathrm{Air}}}{m_{\mathrm{Air}}+m_{\mathrm{EGR}}} W_{\text {Inlet }}, \\
\dot{m}_{\mathrm{EGR}}= & W_{\mathrm{EGR}}-\frac{m_{\mathrm{EGR}}}{m_{\mathrm{Air}}+m_{\mathrm{EGR}}} W_{\text {Inlet }}, \\
\dot{m}_{\mathrm{Exh}}= & W_{\mathrm{Exh}}-W_{\mathrm{Turb}}-W_{\mathrm{EGR}},
\end{aligned}
$$

with

$$
\Psi\left(\frac{p_{1}}{p_{0}}\right)=\left\{\begin{array}{c}
\sqrt{\frac{2 \kappa}{\kappa-1}\left\{\left(\left(\frac{p_{1}}{p_{0}}\right)^{\frac{2}{\kappa}}-\left(\frac{p_{1}}{p_{0}}\right)^{\frac{\kappa+1}{\kappa}}\right)\right\}}, \\
\text { if }\left(\frac{p_{1}}{p_{0}}\right) \geq\left(\frac{2}{\kappa+1}\right)^{\frac{\kappa}{\kappa-1}} \\
\sqrt{\kappa\left(\frac{2}{\kappa+1}\right)^{\frac{\kappa+1}{\kappa-1}}} \text { otherwise, }
\end{array}\right.
$$

$$
W_{\text {Inlet }}=f_{\text {vol }}\left(N_{\text {eng }}, \frac{P_{\text {Inlet }}}{T_{\text {Inlet }} R_{\text {Inlet }}}\right) \frac{N_{\text {eng }} P_{\text {Inlet }}}{T_{\text {Inlet }} R_{\text {Inlet }}} \frac{V_{\text {Eng }}}{120},
$$$$
W_{\mathrm{EGR}}=\frac{A_{\mathrm{EGR}} P_{\mathrm{Exh}}}{\sqrt{R_{\mathrm{Exh}} T_{\mathrm{Exh}}}} \Psi_{\kappa_{\mathrm{Exh}}}\left(\frac{P_{\text {Inlet }}}{P_{\mathrm{Exh}}}\right),
$$

$$
T_{\text {Inlet }}=\frac{P_{\text {Inlet }} V_{\text {Inlet }}}{\left(m_{\text {Air }}+m_{\mathrm{EGR}}\right) R_{\text {Inlet }}},
$$

$$
\begin{aligned}
T_{\mathrm{EGR}} & =\left(\frac{P_{\text {Inlet }}}{P_{\text {Exh }}}\right)^{\frac{\kappa_{\mathrm{Exh}}-1}{\kappa_{\mathrm{Exh}}}} T_{\mathrm{Exh}}, \\
W_{\text {Exh }} & =W_{\text {Inlet }}+W_{\text {Fuel }}, \\
T_{\text {Exh }} & =T_{\text {Inlet }}+\frac{Q_{\mathrm{LHV}} \lambda\left(W_{\text {Fuel }}, N_{\text {Eng }}\right)}{c_{p, \text { Exh }}\left(W_{\text {Inlet }}+W_{\text {Fuel }}\right)}, \\
P_{\text {Exh }} & =\frac{m_{\text {Exh }} T_{\text {Exh }}}{V_{\text {Exh }}}, \\
W_{\text {Turb }} & =\frac{P_{\text {Exh }}}{{\sqrt{T_{\text {Exh }}}}}\left(\frac{P_{\text {Exh }}}{P_{\text {Atm }}}, X_{\mathrm{VGT}}\right), \\
R_{\text {Inlet }} & =\frac{R_{\text {Air }} m_{\text {Air }}+R_{\text {Exh }} m_{\text {EGR }}}{m_{\text {Air }}+m_{\mathrm{EGR}}}, \\
c_{v, \text { Inlet }} & =\frac{c_{v, \text { Air }} m_{\text {Air }}+c_{v, \text { Exh }} m_{\mathrm{EGR}}}{m_{\text {Air }}+m_{\mathrm{EGR}}}, \\
c_{p, \text { Inlet }} & =c_{v, \text { Inlet }}+R_{\text {Inlet }},
\end{aligned}
$$

where $P_{\text {Inlet }}$ is the pressure in the intake manifold. $m_{\text {Air }}$ and $m_{\mathrm{EGR}}$ are respectively the mass of air and EGR-gas in intake manifold. $m_{\text {Exh }}$ represents the mass of exhaust gas in exhaust manifold. The other variables and parameters used in this model are listed in TableB1. The temperature $T_{\mathrm{EGR}}$ is assumed to be constant and equal to $329.436 \mathrm{~K}$. The static functions $f_{\mathrm{vol}}, \lambda$ and $\tau$ are represented as interpolation in lookup tables. Indeed, the modeling step is not evoked in this paper and it has been assumed that the diesel engine model used is well known and all its parameters are correctly identified. However, an interesting work about diesel engines modeling (engines with VGT and EGR) is presented and validated by Wahlstrom and Eriksson (2011).

The system (20)-(23) can be written in the state space form as (1), where the state, known input and output 
vectors and the variables $\rho$ and $v$ are defined as

$$
\begin{aligned}
& x=\left[\begin{array}{llll}
P_{\text {Inlet }} & m_{\text {Air }} & m_{\mathrm{EGR}} & m_{\mathrm{Exh}}
\end{array}\right]^{T} \text {, } \\
& u=\left[\begin{array}{lll}
A_{\mathrm{EGR}} & X_{\mathrm{VGT}} & W_{\text {Fuel }}
\end{array}\right]^{T}, \\
& y=\left[\begin{array}{ll}
P_{\text {Inlet }} & P_{\text {Exh }}
\end{array}\right]^{T} \text {, } \\
& \rho=N_{\text {Eng }} \text {, } \\
& v=\left[\begin{array}{ll}
T_{\mathrm{CAC}} & W_{\mathrm{HFM}}
\end{array}\right]^{T} \text {. }
\end{aligned}
$$

In diesel engines, actuator faults can be any dysfunction on the EGR-valve, in the position of VGT vanes or a fuel leakage. In this paper, the fuel leakage is the only fault considered. However, it is also possible to construct other residuals where each one is sensitive only to one actuator fault (EGR or VGT actuator faults) and insensitive to all other faults. For the fault isolation purpose, only one component of $u$ is considered to be faulty. The other components of $u$ are considered to be unknown inputs $(d)$.

The variables $\rho_{1}$ and $\rho_{2}$ are defined as

$$
\rho_{1}=\frac{\bar{N}_{\text {eng }}-N_{\text {eng }}}{\bar{N}_{\text {eng }}-\underline{N}_{\text {eng }}}, \quad \rho_{2}=\frac{N_{\text {eng }}-\underline{N_{\text {eng }}}}{\bar{N}_{\text {eng }}-\underline{N_{\text {eng }}}},
$$

where $\underline{N}_{\text {eng }}$ and $\bar{N}_{\text {eng }}$ are respectively the minimum and maximum values of the measurable variable $N_{\mathrm{eng}}$.

Matrices $A_{1}$ and $A_{2}$ are given by

$$
\begin{aligned}
A_{1}= & {\left[\begin{array}{cccc}
-\digamma_{1} & 0 & 0 & 0 \\
0 & -\digamma_{1} & 0 & 0 \\
0 & 0 & -\digamma_{1} & 0 \\
0 & \digamma_{1} & \digamma_{1} & 0
\end{array}\right], } \\
A_{2}= & {\left[\begin{array}{cccc}
-\digamma_{2} & 0 & 0 & 0 \\
0 & -\digamma_{2} & 0 & 0 \\
0 & 0 & -\digamma_{2} & 0 \\
0 & \digamma_{2} & \digamma_{2} & 0
\end{array}\right], }
\end{aligned}
$$

with

$$
\begin{aligned}
& \digamma_{1}=\frac{f_{\mathrm{vol}} \underline{N}_{\text {eng }} V_{\text {Eng }}}{120 V_{\text {Inlet }}}, \\
& \digamma_{2}=\frac{f_{\text {vol }} \bar{N}_{\text {eng }} V_{\text {Eng }}}{120 V_{\text {Inlet }}} .
\end{aligned}
$$

Matrices $B_{g}, C, B_{D}, B_{f}$ and $B_{w}$ are expressed as

$$
B_{g}=\left[\begin{array}{cc}
\frac{R_{\text {Air }} c_{p, \text { Air }}}{c_{v, \text { Air }} V_{\text {Inlet }}} & 0 \\
0 & 1 \\
0 & 0 \\
0 & 0
\end{array}\right],
$$

$$
\begin{aligned}
C & =\left[\begin{array}{cccc}
1 & 0 & 0 & 0 \\
0 & 0 & 0 & \frac{R_{\mathrm{Exh}} T_{\mathrm{Exh}}^{\mathrm{avg}}}{V_{\mathrm{Exh}}}
\end{array}\right], \\
B_{d} & =\left[\begin{array}{cc}
\frac{R_{\mathrm{Exh}} c_{p, \mathrm{Exh}} T_{\mathrm{EGR}}}{c_{v, \mathrm{Exh}} V_{\mathrm{Inlet}} \sqrt{R_{\mathrm{Exh}} T_{\mathrm{Exh}}}} & 0 \\
0 & 0 \\
\frac{1}{\sqrt{R_{\mathrm{Exh}} T_{\mathrm{Exh}}}} & 0 \\
\frac{1}{\sqrt{R_{\mathrm{Exh}} T_{\mathrm{Exh}}}} & \frac{-1}{\sqrt{T_{\mathrm{Exh}}}}
\end{array}\right], \\
B_{f} & =\left[\begin{array}{c}
0 \\
0 \\
0 \\
1
\end{array}\right], \\
B_{w}= & {\left[\begin{array}{lll}
0 & 0 & 0
\end{array}\right]^{T}, }
\end{aligned}
$$

with $T_{\text {Exh }}^{\text {avg }}$ being the mean value of the measurable variable $T_{\text {Exh. }}$. In fact, this approximation is chosen in order to decrease the number of variables to be used in observer design, which results in decreasing the number of LMIs to be solved, which is important from a practical point of view. Indeed, the general case when the output equation can be rewritten as

$$
\begin{aligned}
y & =\sum_{j=1}^{n_{\rho}} \varrho_{j} C_{j} x \\
& =C x+\sum_{j=1}^{n_{\rho}} \varrho_{j}\left(C_{j}-C\right) x=C x+D_{w} w,
\end{aligned}
$$

with

$$
D_{w}=\left[\begin{array}{ll}
0 & 1
\end{array}\right]^{T} .
$$

Hence, $w$ is regarded as disturbances which can be rejected using the $H_{\infty}$ performance.

Finally, the functions $g$ and $\varphi$ are given by

$$
g=\left[\begin{array}{ll}
W_{\mathrm{HFM}} T_{\mathrm{CAC}} & W_{\mathrm{HFM}}
\end{array}\right]^{T},
$$

$$
\varphi=\left[\begin{array}{l}
\frac{R_{\text {Inlet }} P_{\text {Inlet }}}{c_{v, \text { Inlet }}} \\
0 \\
\frac{A_{\mathrm{EGR}} m_{\mathrm{Exh}} \Psi_{\kappa_{\mathrm{Exh}}} T_{\mathrm{Exh}}}{\sqrt{R_{\mathrm{Exh}} T_{\mathrm{Exh}}}} \\
-\frac{R_{\mathrm{Exh}} T_{\mathrm{Exh}} m_{\mathrm{Exh}} \tau\left(\frac{P_{\mathrm{Exh}}}{P_{\mathrm{Atm}}}, X_{\mathrm{VGT}}\right)}{V_{\mathrm{Exh}} \sqrt{T_{\mathrm{Exh}}}}
\end{array}\right] .
$$

\section{Simulation results}

5.1. Diagnosis setup. The proposed approach has been tested on a four-cylinder diesel engine model 
running on AMEsim $\AA$ platform in co-simulation with Mathworks Matlab®/Simulink. The geometrical engine characteristics are presented in Table 1.

The co-simulation environment allows us to run two separate models with two different kinds of software at the same time. Both solvers compute independently their respective systems and exchange information with a fixed communication step interval. The co-simulation platform is used to run the engine model on the AMESim part and the diagnosis algorithm (robust observer) on the Simulink part.

Table 1. Geometrical engine characteristics.

\begin{tabular}{|c||c|}
\hline stroke & $86.5 \mathrm{~mm}$ \\
bore & $86.5 \mathrm{~mm}$ \\
connecting rod length & $150 \mathrm{~mm}$ \\
compression ratio & 18 \\
squish height & $0.8 \mathrm{~mm}$ \\
\hline
\end{tabular}

5.2. Model validation. The model described in Section 4 was simulated and compared with validation data obtained by AMEsim by Djemili et al. (2011a). All co-simulation tests have been done on load transients at 1500 rpm, i.e., each load kept for 15 seconds. A typical difference between simulated and measured pressures (AMESim) $P_{\text {Inlet }}$ was observed and the model was shown to have an average error less than $3 \%$, which is acceptable. The source of differences is explained by the following reasoning:

- In the model heat exchangers volume and pipes volumes are neglected.

- The volumetric efficiency $f_{\mathrm{vol}}$ is obtained from a lookup table which differs from the real instantaneous value and depends on the engine operating point. This may lead to errors in real estimation of the gas taken into cylinders.

5.3. Residual generation. Let us first present the numerical values of the variables and parameters used in this work. For our application, we have

$$
\begin{aligned}
& U=10^{-8} \times\left[\begin{array}{cccc}
0 & 0 & -1.5988 & 0 \\
0 & 0 & 0 & -0.8951
\end{array}\right]^{T}, \\
& V=10^{-15} \times\left[\begin{array}{cc}
-0.4441 & 0.0215 \\
-0.4441 & -0.4441
\end{array}\right],
\end{aligned}
$$

and

$$
\begin{array}{ll}
h_{11}^{\max }=-10, & h_{12}^{\max }=776.8324, \\
h_{13}^{\max }=-538.883, & h_{14}^{\max }=1.14192 \times 10^{8}, \\
h_{34}^{\max }=2.2691, & h_{44}^{\max }=-1.5663,
\end{array}
$$

$$
\begin{array}{ll}
h_{11}^{\min }=-15, & h_{12}^{\min }=24.273, \\
h_{13}^{\min }=-2.651 \times 10^{3}, & h_{14}^{\min }=4.959 \times 10^{6}, \\
h_{34}^{\min }=0.0793, & h_{44}^{\min }=-44.8256 .
\end{array}
$$

the rest of $h_{i j}^{\max }$ and $h_{i j}^{\min }$ being zeros.

The scalar factor $Z_{H}$ is equal to $6(4 \times 4-10)$. We obtain, after solving the LMIs (15a)-(15c) using YALMIP, a toolbox for modeling and optimization in Matlab ${ }^{\circledR}$, the following results:

$$
\begin{aligned}
P & =\left[\begin{array}{cccc}
0.0500 & 0 & 0 & 0 \\
0 & 0.0612 & 0 & 0 \\
0 & 0 & 0.0842 & -0.0039 \\
0 & 0 & -0.0039 & 0.0500
\end{array}\right], \\
\bar{Y} & =10^{6} \times\left[\begin{array}{cc}
0 & 0 \\
0 & 0 \\
-3.0296 & -0.1464 \\
0.1152 & 0.0056
\end{array}\right], \\
\bar{K}_{1} & =10^{-7} \times\left[\begin{array}{cc}
1.79 \times 10^{7} & 0 \\
30.62 & -2.08 \\
0 & 0 \\
4.9 & 16.11
\end{array}\right], \\
\bar{K}_{1} & =10^{-7} \times\left[\begin{array}{cc}
1,79 \times 10^{7} & 0 \\
0 & 0 \\
-0.0209 & 0.013 \\
0.2 & 0.16
\end{array}\right] .
\end{aligned}
$$

The value of the attenuation of the disturbance term is chosen as $\gamma_{1,2}=0.0316$. Now, we can easily deduce $Y$ and $K_{k}$ from $P^{-1} \bar{Y}$ and $P^{-1} \bar{K}_{k}$, respectively. The matrix $E$ is found as

$$
E=U+Y V=\left[\begin{array}{cccc}
-1 & 0 & 0 & 0 \\
0 & 0 & 0 & 0
\end{array}\right]^{T}
$$

The observer gain matrices can now be computed and given by

$$
\begin{gathered}
M=\left[\begin{array}{llll}
0 & 0 & 0 & 0 \\
0 & 1 & 0 & 0 \\
0 & 0 & 1 & 0 \\
0 & 0 & 0 & 0
\end{array}\right], \\
L_{1}=10^{-7} \times\left[\begin{array}{cc}
0 & 0 \\
-0.8841 & 0.0007 \\
0.0064 & 0 \\
0.0502 & -0.0001
\end{array}\right], \\
L_{2}=10^{-8} \times\left[\begin{array}{cc}
0 & 0 \\
0 & 0 \\
0.0792 & -0.0001 \\
0.1625 & -0.0007
\end{array}\right],
\end{gathered}
$$




$$
\begin{gathered}
N_{1}=\left[\begin{array}{ccc}
-35.839 & 0.0001 & 0 \\
-0.0001 & -29.2698 & 0 \\
0 & -21.6507 & -14.5833 \\
0 & -468.3496 & -0.0003 \\
& 0 \\
& 380.9507 \\
& -2.7758 \\
& -36.2167
\end{array}\right], \\
N_{2}=\left[\begin{array}{ccc}
-35.8393 & 0 & 0 \\
0 & -29.1667 & 0 \\
0 & 0 & -29.1667 \\
0 & -0.0006 & -0.0006 \\
& 0 \\
& 0.0005 \\
& -3.4134 \\
& -36.1644
\end{array}\right] .
\end{gathered}
$$

Notice that the approach proposed by Chen and Saif (2006) gives no solution since the Lipschitz constant is greater than $10^{8}$. Other methods can be used, or extended to our case, as that proposed by Zemouche and Boutayeb (2009) for estimating the state and unknown input vectors. Unfortunately, this method is computationally demanding since the number of LMIs that should be solved is equal to $N_{L M I}=2^{n \times n_{f}}$, where $n_{f}$ is the number of nonlinearities in the system. So, in our case $N_{L M I}=2^{4 \times 4}=65536$. In addition, it is only proposed for standard systems with a linear time-invariant part $(A x)$. The number of LMIs will largely increase if this method is extended to our LPV case.

The initial conditions for the observer are chosen arbitrarily and given by

$$
z_{0}=\left[\begin{array}{llll}
9 & 0.0004 & 0.0008 & 9 \times 10^{-11}
\end{array}\right]^{T} .
$$

For illustrating the performance of the proposed approach, the unknown input vector $d$, which represents in our case the two other possible actuator faults affecting the EGR-valve and the position of VGT vanes, is taken as

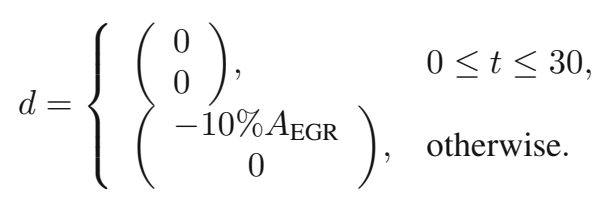

The fault considered is a step-like fault with a magnitude equal to $-30 \%$ of the mass-flow of the injected fuel. This fault is due either to a drop in the pressure in the common rail or a dysfunction of one or more injectors, introduced at $t=10 \mathrm{~s}$ (see (50)),

$$
f= \begin{cases}0, & 0 \leq t \leq 10, \\ -30 \% W_{\text {Fuel }}, & \text { otherwise. }\end{cases}
$$

To show the insensitivity of the residuals to operating point changes and unknown inputs, the following scenario was performed. First, a drift-like change in the engine speed starts at $t=20 \mathrm{~s}$ is introduced (see 3 ). Next, an abrupt change in the $A_{\mathrm{EGR}}$ equivalent to $10 \%$ of the EGR valve effective area, which is computed or fixed by the controller, is considered. Notice that, for the observer design purpose, the minimum and maximum values of $N_{\text {eng }}\left(\underline{N}_{\text {eng }}\right.$ and $\left.\bar{N}_{\text {eng }}\right)$ are taken as 2000 and $4000 \mathrm{rpm}$, respectively.

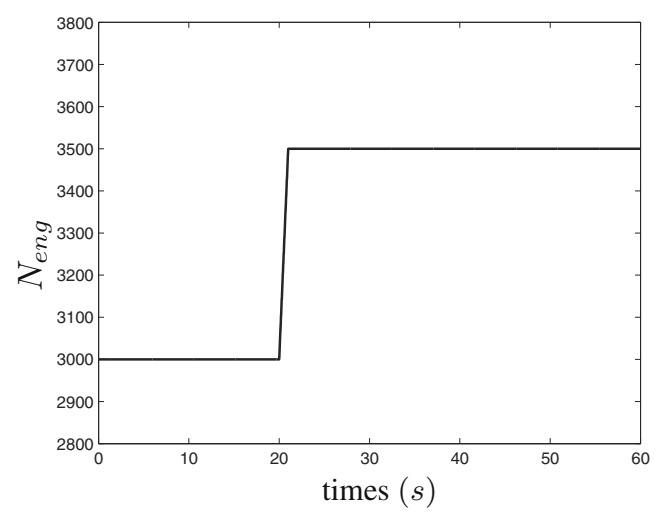

Fig. 2. Engine speed behavior.

The measured inlet and exhaust pressures are shown in Fig 3. The residual behavior is illustrated in Fig. 4. In the nominal case, in the time interval $[0,10] \mathrm{s}$, the residuals $r$ tend asymptotically to zero, as expected. As seen in this figure, the sensitivity of the proposed observer to the fault in the fuel mass flow is well shown from the time instant $t=10 \mathrm{~s}$. Besides, its insensitivity to unknown inputs and operating point changes is well illustrated on the time intervals $[20,30] \mathrm{s}$ and $[30,60] \mathrm{s}$, respectively.

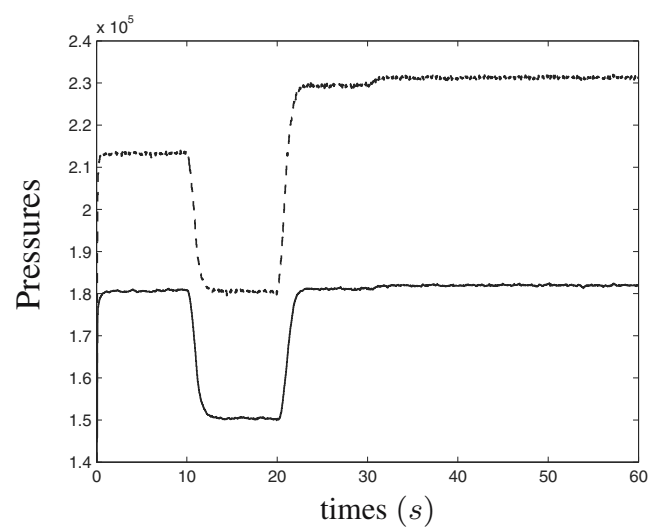

Fig. 3. Measured inlet pressure (continuous line) and exhaust pressure (dashed line). 


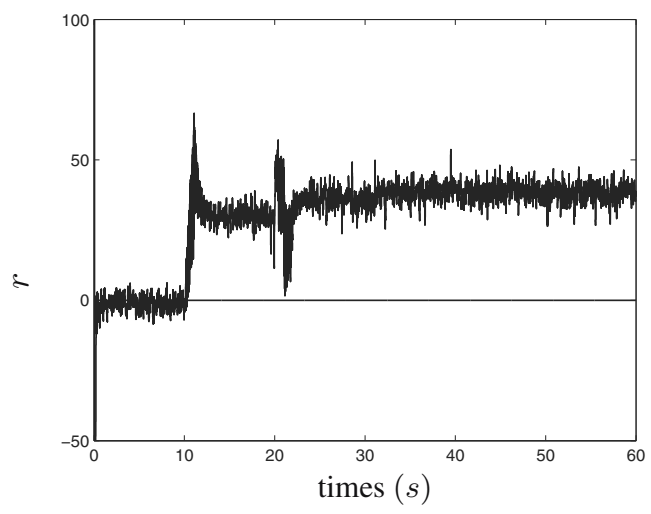

Fig. 4. Residual behavior.

\section{Conclusion}

In this paper, a robust nonlinear unknown input observer for actuator fault detection is proposed. It is developed for a general class of nonlinear systems with a locally or globally bounded Jacobian. Sufficient conditions for the existence of the NUIO are given in terms of matrix inequalities. An $H_{\infty}$ performance index is used to attenuate the disturbances. The developed approach is used successfully to design an NIUO for diesel engines. The performances of the proposed approach are shown using the professional diesel engine simulator AMEsim(LMS) in co-simulation with the Simulink software. A validation of this result with real data issued from the Caterpillar 3126b Engine, located at Sussex University, UK, is a subject of future research.

\section{Acknowledgment}

This work was produced in the framework of SCODECE (Smart COntrol and Diagnosis for Economic and Clean Engine), a European territorial cooperation project part-funded by the European Regional Development Fund (ERDF) through the INTERREG IV A 2 Seas Programme, and the Research Department of the Nord Pas de Calais Region, France.

\section{References}

Albrecht, A., Chauvin, J., Lafossas, F., Potteau, S. and Corde, G. (2006). Development of highly premixed combustion diesel model: From simulation to control design, SAE 2006 World Congress \& Exhibition, Detroit, MI, USA.

CARB (1993). California's OBD-II Regulation (Section 1968.1 Title 13, California Code of Regulations), Resolution 93-40, July 9, pp. 220.7-220.12(h).

Ceccarelli, R., Canudas-de Wit, C., Moulin, P. and Sciarretta, A. (2009). Model-based adaptive observers for intake leakage detection in diesel engines, IEEE American Control Conference (ACC 09), St. Louis, MO, USA, pp. 1128-1133.
Ceccarelli, R., Moulin, P. and Canudas-de Wit, C. (2009). Robust strategy for intake leakage detection in diesel engines, IEEE Control Applications (CCA), Intelligent Control (ISIC), Saint Petersburg, Russia, pp. 340-345.

Chen, W., Khan, A.Q., Abid, M. and Ding, S.X. (2011). Integrated design of observer based fault detection for a class of uncertain nonlinear systems, International Journal of Applied Mathematics and Computer Science 21(3): 423-430, DOI: 10.2478/v10006-011-0031-0.

Chen, W. and Saif, S. (2006). Unknown input observer design for a class of nonlinear systems: An LMI approach, IEEE American Control Conference, Minneapolis, MN, USA, pp. 834-38.

Darouach, M., Zasadzinski, M. and Xu, S. J. (1994). Full-order observers for linear systems with unknown inputs, IEEE Transactions on Automatic Control 39(3): 607-609.

Ding, X., Frank, P.M. and Guo, L. (1990). Nonlinear observer design via extended observer canonical form, Systems and Control Letters 15(4): 313-322.

Djemili, I., Aitouche, A. and Cocquempot, V. (2011a). Adaptive observer for intake leakage detection in diesel engines described by Takagi-Sugeno model, 19th Mediterranean Conference on Control \& Automation (MED 2011), Corfu, Greece, pp. 754-759.

Djemili, I., Aitouche, A. and Cocquempot, V. (2011b). Structural analysis for air path of an automotive diesel engine, IEEE International Conference on Communications, Computing and Control Applications (CCCA'11), Hammamet, Tunisia, pp. 1-6.

Gertler, J., Costin, M., Fang, X. and Hira, R. (1995). Mode based diagnosis for automotive engines: Algorithm development and testing on a production vehicle, IEEE Transactions on Control Systems Technology 3(1): 61-69.

Heywood, J. (1992). Internal Combustion Engine Fundamentals, McGraw-Hill Series in Mechanical Engineering, McGraw-Hill, New York, NY.

Hou, M. and Muller, P.C. (1992). Design of observers for linear systems with unknown inputs, IEEE Transactions on Automatic Control 37(6): 871-874.

Hui, S. and Żak, S.H. (2005). Observer design for systems with unknown inputs, International Journal of Applied Mathematics and Computer Science 15(4): 431-446.

Kao, K. and Moskwa, J. (1995). Turbocharged diesel engine modeling for nonlinear engine control and state estimation ASME Journal of Dynamic Systems, Measurement and Control 117(1): 20-30.

Kudva, P., Viswanadham, N. and Ramakrishna, A. (1980). Observers for linear systems with unknown inputs, IEEE Transactions on Automatic Control 25(1): 113-115.

Nyberg, M. (2002). Model-based diagnosis of an automotive engine using several types of fault models, IEEE Transactions on Control Systems Technology 10(5): 679-689.

Nyberg, M. and Perkovic, A. (1998). Model based diagnosis of leaks in the air-intake system of an SI-engine, International Congress \& Exposition, Detroit, MI, USA. 
Nyberg, M. and Sutte, T. (2004). Model based diagnosis of the air path of an automotive diesel engine, Control Engineering Practice 12(5): 513-525.

Phanomchoeng, G., Rajamani, R. and Piyabongkarn, D. (2011). Nonlinear observer for bounded Jacobian systems, with applications to automotive slip angle estimation, IEEE Transactions on Automatic Control 56(5): 1163-1170.

Saif, M. (1993). A disturbance accommodating estimator for bilinear systems, IEEE American Control Conference (ACC 93), San Francisco, CA, USA, pp. 945-949.

Seliger, R. and Frank, P.M. (1991). Robust component fault detection and isolation in nonlinear dynamic systems using nonlinear unknown input observers, Proceedings of the IFAC/IMACS Symposium SAFEPROCESS, Baden-Baden, Germany, pp. 313-318.

Wahlstrom, J. and Eriksson, L. (2011). Modelling diesel engines with a variable-geometry turbocharger and exhaust gas recirculation by optimization of model parameters for capturing non-linear system dynamics, Proceedings of the Institution of Mechanical Engineers, Part D: Journal of Automobile Engineering 225(7): 960-986.

Yaz, E. and Azemi, A. (1998). Actuator fault detection and isolation in nonlinear systems using LMIs and LMEs, IEEE American Control Conference (ACC 98), Philadelphia, PA, USA, Vol. 3, pp. 1590-1594.

Zemouche, A. and Boutayeb, M. (2009). Sobolev norms-based state estimation and input recovery for a class of nonlinear systems. design and experimental results, IEEE Transactions on Signal Processing 57(3): 1021-1029.

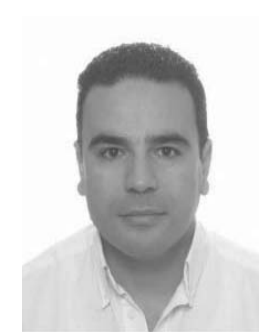

Boulaid Boulkroune received the M.Sc. degree in automatic control from the National Polytechnic Institute of Lorraine, France, in 2004 and the Ph.D. degree in automatic control from Henri Poincaré Nancy I University, France, in 2008. He was a postdoctoral researcher at the Department of Control Engineering and System Analysis, ULB, Brussels, Belgium, till 2011. Currently, he is a postdoctoral researcher at HEI, Engineer School, Lille, France. His research interests are in the areas of process control, diesel engines, fault diagnosis, state estimation, and fault detection and isolation.

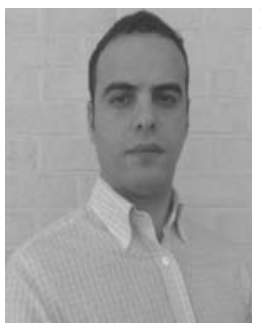

Issam Djemili received his engineer's degree in automatic control from Annaba University, Algeria, in 2008, his master's degree from the National Polytechnic Institute of Lorraine (INPL), France, in 2009, and his Ph.D. from Lille 1 University, France, in 2012. He is now working as a consultant for various companies in automotive industry. His research interests include fault diagnosis and fault tolerant control.

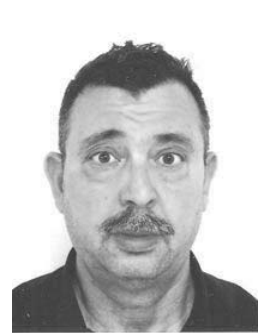

Abdel Aitouche is a professor in the Graduate School of Engineering, France. He is a researcher at LAGIS-CNRS UMR 8219: Automatic Control, Computer Science and Signal Processing Laboratory (associated with the CNRS, French National Center for Scientific Research). He is a member of several scientific societies such the IEEE CSS, IEEE RAS and MCA (Mediterranean Control Society). Aitouche's research interests concern model based fault detection and diagnosis, fault tolerant control, robust nonlinear observers and control. The application domains are mainly transportation, process engineering, fermented process, diesel engine and renewable energy (wind, PV and fuel cell). He has authored several publications in this area.

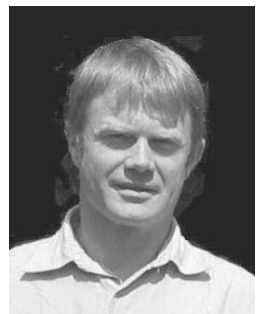

Vincent Cocquempot received the Ph.D. degree in automatic control from the Lille University of Sciences and Technologies in 1993. He is currently a full professor of automatic control and computer science at Lille 1 University, France. $\mathrm{He}$ is a researcher of LAGIS-CNRS UMR 8219: Automatic Control, Computer Science and Signal Processing Laboratory of Lille 1 University, and the head of the team on fault tolerant systems in this laboratory. His research interests include robust on-line fault detection and isolation for uncertain dynamical nonlinear systems and fault tolerant control for hybrid dynamical systems. He is a member of the IFAC Technical Committee on Fault Detection, Supervision, and Safety of Technical Processes.

\section{Appendix A}

\section{Proof of Theorem 2}

Using Theorem 11 the nonlinear term $\tilde{\varphi}$ can be written as

$$
\begin{aligned}
& \tilde{\varphi}=\left[\left(\sum_{i, j=1}^{n, n} H_{i j}^{\max } \delta_{i j}^{\max }\right)+\left(\sum_{i, j=1}^{n, n} H_{i j}^{\min } \delta_{i j}^{\min }\right)\right] \bar{e} \\
& \delta_{i j}^{\max }, \delta_{i j}^{\min } \geq 0, \quad \delta_{i j}^{\max }+\delta_{i j}^{\min }=1 .
\end{aligned}
$$

To simplify the form of the final result as in the work of Phanomchoeng et al. (2011), the term

$$
\sum_{i, j=1}^{n, n}\left(\delta_{i j}^{\max }+\delta_{i j}^{\min }\right)
$$

must be scaled to one. If all the terms in $\frac{\partial \varphi_{i}}{\partial x_{j}}$ are not zero, then the scaling factor $Z_{H}$ can be computed by

$$
\sum_{i, j=1}^{n, n}\left(\delta_{i j}^{\max }+\delta_{i j}^{\min }\right)=n \times n=Z_{H},
$$




$$
\frac{\sum_{i, j=1}^{n, n}\left(\delta_{i j}^{\max }+\delta_{i j}^{\min }\right)}{Z_{H}}=1 .
$$

Then, A1 is rewritten as

$$
\begin{aligned}
& \tilde{\varphi}=\left[\left(\sum_{i, j=1}^{n, n} \bar{H}_{i j}^{\max } \bar{\delta}_{i j}^{\max }\right)+\left(\sum_{i, j=1}^{n, n} \bar{H}_{i j}^{\min } \bar{\delta}_{i j}^{\min }\right)\right] \bar{e}, \\
& \bar{\delta}_{i j}^{\max }, \bar{\delta}_{i j}^{\min } \geq 0 \\
& \bar{\delta}_{i j}^{\max }+\bar{\delta}_{i j}^{\min }=\frac{1}{Z_{H}} \\
& \sum_{i, j=1}^{n, n}\left(\bar{\delta}_{i j}^{\max }+\bar{\delta}_{i j}^{\min }\right)=1
\end{aligned}
$$

where

- $\bar{H}_{i j}^{\max }=Z_{H} H_{i j}^{\max }$ and $\bar{H}_{i j}^{\min }=Z_{H} H_{i j}^{\min }$,

- $\bar{\delta}_{i j}^{\max }=\delta_{i j}^{\max } / Z_{H}$ and $\bar{\delta}_{i j}^{\min }=\delta_{i j}^{\min } / Z_{H}$.

Based on $\mathrm{A} 3$, the function $\Gamma$ becomes

$$
\begin{aligned}
\Gamma= & {\left[\begin{array}{c}
\bar{e}^{T} \\
w^{T} \\
\dot{w}^{T}
\end{array}\right]\left[\begin{array}{ccc}
\Lambda & P\left(K D_{w}-M B_{w}\right) & -P E D_{w} \\
(*) & -\mu I_{n_{w}} & 0 \\
(*) & (*) & -\mu I_{n_{w}}
\end{array}\right] } \\
& \times\left[\begin{array}{c}
\bar{e}^{T} \\
w^{T} \\
\dot{w}^{T}
\end{array}\right],
\end{aligned}
$$

where $\mu=\gamma_{1,2}^{2}$,

$$
\begin{aligned}
\Lambda= & \sum_{i, j=1}^{n, n} \bar{\delta}_{i j}^{\max } \Xi_{i j}^{\max }+\sum_{i, j=1}^{n, n} \bar{\delta}_{i j}^{\min } \Xi_{i j}^{\min }, \\
\Xi_{i j}^{\max }= & \left(M A-K C+M \bar{H}_{i j}^{\max }\right)^{T} P \\
& +\left(M A-K C+M \bar{H}_{i j}^{\max }\right)+I_{n_{x}}, \\
\Xi_{i j}^{\min }= & \left(M A-K C+M \bar{H}_{i j}^{\min }\right)^{T} P \\
& +P\left(M A-K C+M \bar{H}_{i j}^{\min }\right)+I_{n_{x}},
\end{aligned}
$$

the negativity of $\Gamma$ being ensured if

$$
\sum_{i, j=1}^{n, n} \bar{\delta}_{i j}^{\max } \underbrace{\left[\begin{array}{ccc}
\Xi_{i j}^{\max } & P\left(K D_{w}-M B_{w}\right) & -P E D_{w} \\
(*) & -\mu I_{n_{w}} & 0 \\
(*) & (*) & -\mu I_{n_{w}}
\end{array}\right]}_{M_{i j}^{\max }}
$$

$$
+\sum_{i, j=1}^{n, n} \bar{\delta}_{i j}^{\min } \underbrace{\left[\begin{array}{ccc}
\Xi_{i j}^{\min } & P\left(K D_{w}-M B_{w}\right) & -P E D_{w} \\
(*) & -\mu I_{n_{w}} & 0 \\
(*) & (*) & -\mu I_{n_{w}}
\end{array}\right]}_{M_{i j}^{\min }}<0 .
$$

Since we have $\bar{\delta}_{i j}^{\max }, \quad \bar{\delta}_{i j}^{\min } \geq 0$, the previous equation is equivalent to

$$
M_{i j}^{\max }<0 \text { and } M_{i j}^{\min }<0 .
$$

By substituting the matrices $A, L$ and $K$ by their expressions, A5 can be written as follows:

$$
\sum_{k=1}^{n_{\rho}} \rho_{k} M_{i j k}^{\max }<0, \quad \sum_{k=1}^{n_{\rho}} \rho_{k} M_{i j k}^{\min }<0,
$$

where

$$
\begin{aligned}
M_{i j k}^{\max } & =\left[\begin{array}{ccc}
\Xi_{i j k}^{\max } & P\left(K_{k} D_{w}-M B_{w}\right) & -P E D_{w} \\
(*) & -\mu I_{n_{w}} & 0 \\
(*) & (*) & -\mu I_{n_{w}}
\end{array}\right], \\
M_{i j k}^{\min } & =\left[\begin{array}{ccc}
\Xi_{i j k}^{\min } & P\left(K_{k} D_{w}-M B_{w}\right) & -P E D_{w} \\
(*) & -\mu I_{n_{w}} & 0 \\
(*) & (*) & -\mu I_{n_{w}}
\end{array}\right],
\end{aligned}
$$

with

$$
\begin{aligned}
\Xi_{i j k}^{\max }= & \left(M A_{k}-K_{k} C+M \bar{H}_{i j}^{\max }\right)^{T} P \\
& +P\left(M A_{k}-K_{k} C+M \bar{H}_{i j}^{\max }\right)+I_{n_{x}}, \\
\Xi_{i j k}^{\min }= & \left(M A_{k}-K_{k} C+M \bar{H}_{i j}^{\min }\right)^{T} P \\
& +P\left(M A_{k}-K_{k} C+M \bar{H}_{i j}^{\min }\right)+I_{n_{x}} .
\end{aligned}
$$

Since $\rho_{k}>0, \quad \forall k=1, \ldots, n_{\rho}$, the condition (A6) holds if

$$
M_{i j k}^{\max }<0, \quad M_{i j k}^{\min }<0,
$$

$\forall i=1, \ldots, n, j=1, \ldots, n$ and $k=1, \ldots, n_{\rho}$.

From $(8 \mathrm{c}), M$ can be written as

$$
M=I_{n_{x}}+U C+Y V C .
$$

To obtain the LMIs 15 - $15 b$, it suffices to substitute the formula of $M$ in A7 and to take $\bar{K}_{k}=$ $P K_{k}$ and $\bar{Y}=P Y$. This ends the proof. 


\section{Appendix B}

\section{Nomenclature}

Table B1. Variables used in the engine model.

\begin{tabular}{|c|c|c|}
\hline Symbol & Quantity & Unit \\
\hline$P_{\text {Inlet }}$ & Pressure in intake manifold & $\mathrm{Pa}$ \\
\hline$V_{\text {Inlet }}$ & Volume of intake manifold & $\mathrm{m}^{3}$ \\
\hline$R_{\text {Air }}$ & Gas constant of air & $J \cdot(\mathrm{Kg} \cdot \mathrm{K})$ \\
\hline$c_{p, \text { Air }}$ & $\begin{array}{l}\text { Specific heat at const. pres. } \\
\text { of air }\end{array}$ & $\mathrm{J} \cdot(\mathrm{Kg} \cdot \mathrm{K})$ \\
\hline$c_{v, \text { Air }}$ & $\begin{array}{l}\text { Specific heat at const. vol. } \\
\text { of air }\end{array}$ & $\mathrm{J} \cdot(\mathrm{Kg} \cdot \mathrm{K})$ \\
\hline$R_{\text {Exh }}$ & $\begin{array}{l}\text { Gas constant of exhaust gas } \\
\text { of exhaust gas }\end{array}$ & $\mathrm{J} \cdot(\mathrm{Kg} \cdot \mathrm{K})$ \\
\hline$c_{p, \mathrm{Exh}}$ & $\begin{array}{l}\text { Specific heat at const. pres. } \\
\text { of exhaust gas }\end{array}$ & $\mathrm{J} \cdot(\mathrm{Kg} \cdot \mathrm{K})$ \\
\hline$c_{v, \text { Exh }}$ & $\begin{array}{l}\text { Specific heat at const. vol. } \\
\text { of exhaust gas }\end{array}$ & $\mathrm{J} \cdot(\mathrm{Kg} \cdot \mathrm{K})$ \\
\hline$R_{\text {Inlet }}$ & Gas constant in intake manifold & $\mathrm{J} \cdot(\mathrm{Kg} \cdot \mathrm{K})$ \\
\hline$c_{p, \text { Inlet }}$ & $\begin{array}{l}\text { Specific heat at const. pres. } \\
\text { in intake manifold }\end{array}$ & $\mathrm{J} \cdot(\mathrm{Kg} \cdot \mathrm{K})$ \\
\hline$c_{v, \text { Inlet }}$ & $\begin{array}{l}\text { Specific heat at const. vol. } \\
\text { in intake manifold }\end{array}$ & $\mathrm{J} \cdot(\mathrm{Kg} \cdot \mathrm{K})$ \\
\hline$\kappa$ & Ratio of specific heats & $\mathrm{c}_{p} / \mathrm{c}_{v}$ \\
\hline$W_{\mathrm{HFM}}$ & $\begin{array}{l}\text { Air mass-flow past air } \\
\text { mass-flow sensor }\end{array}$ & $\mathrm{kg} \cdot \mathrm{s}^{-1}$ \\
\hline$T_{\mathrm{CAC}}$ & $\begin{array}{l}\text { Temperature of air after } \\
\text { charge-air cooler }\end{array}$ & $\mathrm{K}$ \\
\hline$W_{\mathrm{EGR}}$ & $\begin{array}{l}\text { EGR mass-flow into intake } \\
\text { manifold }\end{array}$ & $\mathrm{Kg} \cdot \mathrm{s}^{-1}$ \\
\hline$T_{\mathrm{EGR}}$ & $\begin{array}{l}\text { Temperature of EGR gas- } \\
\text { flow into i.m }\end{array}$ & K \\
\hline$W_{\text {Inlet }}$ & Mass-flow into engine inlet-ports & $\mathrm{kg} \cdot \mathrm{s}^{-1}$ \\
\hline$T_{\text {Inlet }}$ & $\begin{array}{l}\text { Temperature in intake } \\
\text { manifold }\end{array}$ & K \\
\hline$m_{\text {Air }}$ & Mass of air in intake manifold & $\mathrm{Kg}$ \\
\hline$m_{\mathrm{EGR}}$ & Mass of EGR-gas in intake manifold & $\mathrm{kg}$ \\
\hline$W_{\text {Exh }}$ & $\begin{array}{l}\text { Exhaust mass-flow into } \\
\text { exhaust manifold }\end{array}$ & $\mathrm{kg} \cdot \mathrm{s}^{-1}$ \\
\hline$m_{\text {Exh }}$ & $\begin{array}{l}\text { Mass of exhaust gas in } \\
\text { exhaust manifold }\end{array}$ & $\mathrm{kg}$ \\
\hline$P_{\text {Exh }}$ & Pressure in exhaust manifold & $\mathrm{Pa}$ \\
\hline$T_{\text {Exh }}$ & $\begin{array}{l}\text { Temperature in exhaust } \\
\text { manifold }\end{array}$ & K \\
\hline$A_{\mathrm{EGR}}$ & Effective area of EGR valve & $\mathrm{m}^{2}$ \\
\hline$V_{\text {Eng }}$ & Engine displacement & $\mathrm{m}^{3}$ \\
\hline$N_{\text {Eng }}$ & Engine speed & $\min ^{-1}$ \\
\hline$W_{\text {Fuel }}$ & Mass-flow of injected fuel & $\mathrm{kg} \cdot \mathrm{s}^{-1}$ \\
\hline$Q_{\mathrm{LHV}}$ & Lower heating value & $\mathrm{J} \cdot \mathrm{kg}^{-1}$ \\
\hline$V_{\text {Exh }}$ & Volume of exhaust manifold & $\mathrm{m}^{3}$ \\
\hline$p_{\text {Atm }}$ & Atmospheric pressure & $\mathrm{Pa}$ \\
\hline$W_{\text {Turb }}$ & $\begin{array}{l}\text { Exhaust mass-flow past } \\
\text { turbine }\end{array}$ & $k g \cdot s^{-1}$ \\
\hline$X_{\mathrm{VGT}}$ & Position of VGT vanes & $\%$ \\
\hline
\end{tabular}

Received: 18 April 2012

Revised: 21 January 2013

Re-revised: 8 April 2013 